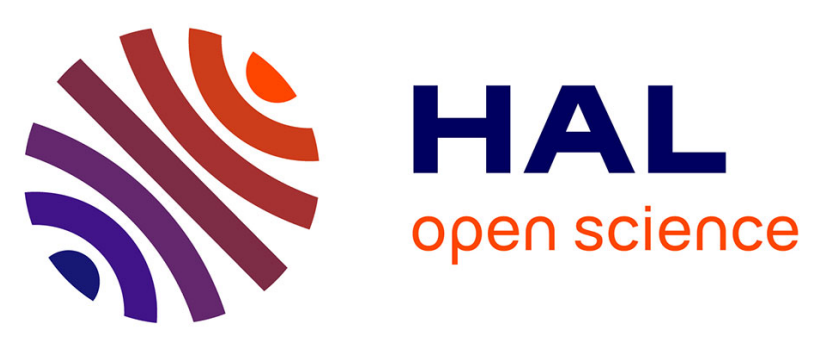

\title{
A PGSE-NMR Study of Molecular Self-Diffusion in Lamellar Phases Doped with Polyoxometalates
}

Andreas S. Poulos, Doru Constantin, Patrick Davidson, Marianne

Impéror-Clerc, Patrick Judeinstein, Brigitte Pansu

\section{- To cite this version:}

Andreas S. Poulos, Doru Constantin, Patrick Davidson, Marianne Impéror-Clerc, Patrick Judeinstein, et al.. A PGSE-NMR Study of Molecular Self-Diffusion in Lamellar Phases Doped with Polyoxometalates. Journal of Physical Chemistry B, 2009, 114 (1), pp.220-227. 10.1021/jp909058d . hal-00441709

\author{
HAL Id: hal-00441709 \\ https://hal.science/hal-00441709
}

Submitted on 3 Feb 2010

HAL is a multi-disciplinary open access archive for the deposit and dissemination of scientific research documents, whether they are published or not. The documents may come from teaching and research institutions in France or abroad, or from public or private research centers.
L'archive ouverte pluridisciplinaire HAL, est destinée au dépôt et à la diffusion de documents scientifiques de niveau recherche, publiés ou non, émanant des établissements d'enseignement et de recherche français ou étrangers, des laboratoires publics ou privés. 


\section{A PGSE-NMR study of molecular self-diffusion in lamellar phases doped with polyoxometalates}

Andreas S. Poulos ${ }^{1}$, Doru Constantin ${ }^{1}$, Patrick Davidson ${ }^{1, *}$, Marianne Impéror ${ }^{1}$, Patrick Judeinstein $^{1,2}$, Brigitte Pansu ${ }^{1}$

${ }^{1}$ Laboratoire de Physique des Solides, UMR 8502, Université Paris-Sud, CNRS, 91405 Orsay, France

${ }^{2}$ Institut de Chimie Moléculaire et des Matériaux d'Orsay, UMR 8182, Université ParisSud, CNRS, 91405 Orsay, France

* Author for correspondence; davidson@1ps.u-psud.fr 
Abstract. Using pulsed gradient spin-echo NMR, we studied molecular self-diffusion in aligned samples of a hybrid lyotropic lamellar $\mathrm{L}_{\alpha}$ phase. This composite organic-inorganic material was obtained by doping the lamellar phase of the non-ionic surfactant Brij-30 with the $\left[\mathrm{PW}_{12} \mathrm{O}_{40}\right]^{3-}$ polyoxometalate (POM). Both water and POM self-diffusion display a large anisotropy as diffusion is severely restricted along the normal to the bilayers. Water diffusion in planes parallel to the bilayers does not depend on the POM concentration but depends on the lamellar period, which is due to a variable fraction of "bound" water molecules. POM diffusion in the hybrid $\mathrm{L}_{\alpha}$ phase is almost two orders of magnitude slower than in aqueous solution. Moreover, it is not at all affected by the thickness of the aqueous medium separating the bilayers. This proves that the POM nanoparticles do not freely diffuse in the inter-bilayer aqueous space but adsorb onto the PEG brushes that cover both sides of the surfactant bilayers.

Keywords: polyoxometalates, lyotropic liquid crystals, non-ionic surfactants, diffusion, PGSENMR 


\section{Introduction}

Organic-inorganic hybrid systems presently raise much interest in materials science because they can potentially combine the structural and electronic properties of inorganic materials with the auto-assembly properties of organic molecules. ${ }^{1-6}$ In this context, polyoxometalates, ${ }^{7-9}$ thanks to their outstanding electronic properties, are inorganic building blocks that are becoming increasingly popular in soft-condensed matter studies. ${ }^{10-23}$ Being negatively charged, they are most often associated to cationic surfactants, via ionic self-assembly processes. In a previous study, we formulated a hybrid system where the inorganic component is the $\left[\mathrm{PW}_{12} \mathrm{O}_{40}\right]^{3-}$ polyoxometalate (POM) anion (fig. 1a) and the organic component is a lyotropic lamellar $\mathrm{L}_{\alpha}$ phase of the non-ionic surfactant Brij 30 (fig. 1b). ${ }^{24}$ With this approach, a weak and non-specific POM-surfactant interaction was expected. Indeed, up to $18 \mathrm{w} \%$ POMs could thus be incorporated into the mesophase. The resulting POM-doped lamellar phase was shown to have retained the well-known photochemical properties of the polyoxometalate anions. At the same time though, the hybrid system still displays the anisotropy and the viscoelasticity of the $\mathrm{L}_{\alpha}$ phase.

The phase diagram (figure 1c) of this system has been studied in detail and displays a wide domain in which the samples are in the $\mathrm{L}_{\alpha}$ phase. ${ }^{24}$ The POM-doped $\mathrm{L}_{\alpha}$ phase can be thought of as stacks of fluid surfactant bilayers separated by aqueous regions were the hydrophilic POMs are located. However, the electron density profile of the $\mathrm{L}_{\alpha}$ phase, determined by small-angle X-ray scattering measurements, ${ }^{24}$ suggests that the POMs are partially localized at the surface of the bilayers. This suggests an interaction stronger than expected, which in principle should affect the POM dynamics. To address this issue, we have used the pulsed gradient spin-echo NMR (PGSE 
NMR) technique ${ }^{25}$ that has been used extensively to study lyotropic phases, usually by measuring the self-diffusion coefficient of water molecules. ${ }^{26}$ This method can indeed provide information on the translational dynamics of the molecules and therefore about the structure of the lyotropic phase. In the present system, we measured not only the diffusion coefficient of water but also that of the confined POMs in order to demonstrate their interaction with the surfactant bilayers.

\section{Experimental}

\subsection{Sample preparation}

\subsubsection{Materials}

The surfactant Brij 30 was purchased from Sigma-Aldrich and used without any further purification. It consists mostly of $\mathrm{C}_{12} \mathrm{EO}_{4}$, along with a smaller amount of homologous $\mathrm{C}_{\mathrm{m}} \mathrm{EO}_{\mathrm{n}}$ molecules. Its phase diagram is very similar to that of pure $\mathrm{C}_{12} \mathrm{EO}_{4}{ }^{27}$ At room temperature, and for concentrations between 25-85 wt\%, aqueous solutions of Brij 30 form a lamellar $\left(\mathrm{L}_{\alpha}\right)$ lyotropic mesophase. By changing the surfactant concentration, the lamellar periodicity can be varied between 5 and $12 \mathrm{~nm}$, so that the inter-membrane distance (i.e. the thickness of the aqueous layers) can be tuned between 1.5 and $8.5 \mathrm{~nm}$.

Phosphotungstic acid hydrate $\left(\mathrm{H}_{3} \mathrm{PW}_{12} \mathrm{O}_{40} \bullet \mathrm{xH}_{2} \mathrm{O}\right)$ was purchased from Sigma-Aldrich and used without any further purification (Purity $99.995 \%$ ). The acid is completely dissociated in water, giving $\left[\mathrm{PW}_{12} \mathrm{O}_{40}\right]^{3-}$ polyoxometalate particles $(\mathrm{POMs})$ and $\mathrm{H}^{+}$. The mass fraction of hydration water, determined by drying at $200^{\circ} \mathrm{C}$, was found to be approximately $15 \%$, which 
corresponds well with the value $(\sim 20 \%)$ already reported in literature. ${ }^{28}$ The POM diameter is $1.1 \mathrm{~nm}^{29}$

\subsubsection{Preparation of POM solutions}

Aqueous POM solutions were prepared by dissolving a known quantity of phosphotungstic acid powder in distilled water. Taking into account the hydration water, the POM mass fraction in the final solution is: $m_{P O M}=0.85 M_{0} /\left(M_{0}+M_{w}\right)$ where $M_{0}$ and $M_{\mathrm{w}}$ are respectively the masses of phosphotungstic acid hydrate and water added.

The POM volume fraction of the final solution $\left(\phi_{P O M}\right)$ is given by:

$$
\phi_{P O M}=\frac{0.85 M_{0} / \rho_{P O M}}{0.85 M_{0} / \rho_{P O M}+\left(0.15 M_{0}+M_{w}\right) / \rho_{w}}
$$

where $\rho_{P O M}$ and $\rho_{\mathrm{w}}$ are the densities of single POM anions and water respectively. The density of a single $\left[\mathrm{PW}_{12} \mathrm{O}_{40}\right]^{3-}$ anion is $\rho_{P O M}=6.98 \mathrm{~g} / \mathrm{mL}$, using the POM volume reported by Pope. ${ }^{29}$ Solutions with a maximum POM volume fraction of $10 \%$ were prepared.

\subsubsection{Preparation of POM-doped $\mathrm{L}_{\alpha}$ phases}

All POM-doped samples are identified by their surfactant volume fraction $\phi_{\text {Surf }}=V_{\text {Surf }} / \mathrm{V}$ where $V_{\text {Surf }}$ is the volume of added surfactant and $V$ is the overall sample volume, and their POM volume fraction in the aqueous medium, $\phi_{\text {POM. }}$. In principle, for $\mathrm{L}_{\alpha}$ samples, $\phi_{\text {Surf }}$ defines the lamellar periodicity and $\phi_{P O M}$ controls the average distance between the POMs within a water layer. 
All samples were prepared by simple mixing of the surfactant Brij 30 (liquid at room temperature) with a POM solution of known $\phi_{P O M}$. The surfactant was weighed in first. $V_{\text {Surf }}$ was calculated based on the density of Brij $30(0.95 \mathrm{~g} / \mathrm{mL})$. To prepare a sample with a given $\phi_{\text {Surf }}$, the appropriate volume of $\mathrm{POM}$ solution, $V_{a q}$, was calculated using a simple dilution law: $V_{a q}=\left(\frac{1}{\phi_{\text {surf }}}-1\right) V_{\text {surf }}$. The correct volume of POM solution was added using a precision micropipette and also weighed to check against the known density of each solution. The POM volume fraction of the added solution is also the $\phi_{P O M}$ of the sample.

After adding all the components, the samples were sealed with parafilm and mixed with a vortexer. Finally, they were centrifuged $(100 \mathrm{~g}, 5 \mathrm{mins})$ to collect all material at the bottom of the tube. The samples were stored inside a cupboard to avoid exposure to light and were left to stand for one week before any experiments.

In the samples used for NMR experiments, $25 \%$ of the water was replaced by deuterated water, which was found to have no effect on the phase diagram of the system.

\subsubsection{Sample alignment}

Without any special treatment, the lamellar samples in the NMR tubes present a partially aligned texture that complicates the interpretation of the PGSE-NMR data. The easiest way to obtain reliable data for anisotropic diffusion of mobile species inside $\mathrm{L}_{\alpha}$ phases is to use macroscopically aligned samples. The alignment procedure consists of heating the sample, held in a NMR tube inside the spectrometer, to the isotropic phase and then cooling it back slowly to the lamellar phase. Indeed, when the mesophase is submitted to a magnetic field, these non-ionic 
surfactant molecules are known to align with their long axes perpendicular to the field. ${ }^{30}$ Therefore, when the lamellar phase is reformed in-situ in the NMR (vertical) field, the membranes align with the director perpendicular to the applied field (and hence perpendicular to the axis of the NMR tube.) This results in a 2-D orientational distribution of lamellar domains ("2-D powder") with directors in the horizontal plane.

The preparation of "true" single domains of POM-doped $\mathrm{L}_{\alpha}$ phase was achieved by using flat, $100 \mu \mathrm{m}$ thick, optical glass capillaries (VitroCom Inc., Mountain Lakes, NJ). Each sample was heated to above the lamellar-to-isotropic transition temperature. Then, the temperature was slowly $(\approx 0.1 \mathrm{~K} / \mathrm{min}$ ) lowered back to room temperature. The lamellar phase domains that nucleate are aligned with their bilayers parallel to the flat faces of the capillary (homeotropic anchoring). These domains then grow to span the whole width of the capillary. However, the amount of material is here much smaller $\left(\sim 2 \mathrm{~mm}^{3}\right)$ so that stacks of ten such capillaries had to be introduced in an NMR tube to perform the PGSE-NMR experiments. Even so, the small amount of material did not allow us to study POM diffusion; only water diffusion could be examined in this configuration.

\subsection{Experimental techniques}

\subsubsection{Pulsed-field spin echo NMR (PGSE-NMR)}

The PGSE-NMR experiments were performed on a Bruker Avance NMR 400 spectrometer with a broad-band probe equipped with three-axis gradient coils without field/frequency lock control. The maximum gradient values for the three perpendicular directions were: $\mathrm{G}_{\mathrm{z}}=45$ 
G.cm ${ }^{-1}, G_{x}=G_{y}=38$ G.cm ${ }^{-1}$, $\mathrm{z}$ being the direction of the constant field. Temperature was controlled within the $300-360 \mathrm{~K}$ range with a Bruker BVT3000 system ( $\pm 1 \mathrm{~K}$ regulation). Temperature calibration was performed before each set of measurements by using a reference ethylene glycol sample.

Self-diffusion was measured with the pulsed field gradient stimulated echo and LED sequence using 2 spoil gradients. ${ }^{31}$ Self-diffusion coefficients were determined from the classical Stejskal-Tanner equation:

$$
\ln \left(I / I_{0}\right)=-D G^{2} \gamma^{2} \delta^{2}(\Delta-\delta / 3)
$$

where $\mathrm{G}$ is the magnitude of the two gradient pulses, $\Delta$ is the time interval between these pulses and $\delta$ is their duration, $\gamma$ is the gyromagnetic ratio of the nucleus under study and $I$ and $I_{0}$ are the integrated intensities of the signals obtained respectively with and without gradient pulses. ${ }^{25}$ The magnitude of the pulsed field gradient was varied between 0 and the maximum available value in 8,10 or 16 steps; the diffusion time $\Delta$ between two pulses was fixed at $50 \mathrm{~ms}$ and $1000 \mathrm{~ms}$ for ${ }^{1} \mathrm{H}$ and ${ }^{31} \mathrm{P}$ respectively, and the pulse duration $\delta$ was set between 3 and $12 \mathrm{~ms}$, depending on the diffusion coefficient to be measured. For the ${ }^{1} \mathrm{H}$ PGSE-NMR experiments, 16 scans were added at each step, with a relaxation delay of $8 \mathrm{~s}$.

In order to prepare the ${ }^{31} \mathrm{P}$ PGSE-NMR experiments, the $\mathrm{T}_{1}$ relaxation times were first measured by standard techniques and found to be around 20 s. Therefore, relaxation delays of $120 \mathrm{~s}$ were used. Besides, the low POM concentration inside these materials requires a minimum of 80 scans for each step of the PGSE measurements to reach an acceptable signal/noise ratio and dependable diffusion coefficient values. Then, the total measurement time to measure a diffusion coefficient in one direction is at least 8 hours. $\pi / 2$ pulse widths were calibrated to 6 and $21 \mathrm{~ms}$ for ${ }^{1} \mathrm{H}$ and ${ }^{31} \mathrm{P}$ nuclei respectively. 
The diffusion coefficient in anisotropic media can be represented as a tensor. In phases with cylindrical symmetry (e.g. nematic or $\mathrm{L}_{\alpha}$ ), only two of its diagonal elements are independent. For a lamellar phase, the normal to the layers, also called director $\mathbf{n}$, is the axis of symmetry; $D_{/ /}$is the coefficient of diffusion parallel to $\mathbf{n}$ (and perpendicular to the lamellae), and $D_{\perp}$ is the coefficient of diffusion perpendicular to $\mathbf{n}$ (and parallel to the lamellae).

For diffusion that is not parallel to one of the main axes of the phase, the relevant diffusion coefficient is a linear combination of $D_{/ /}$and $D_{\perp}$. For a lamellar phase single domain whose director makes an angle $\theta$ with the applied gradient, the diffusion coefficient can be written as:

$$
D_{\theta}=D_{/ /} \cos ^{2} \theta+D_{\perp} \sin ^{2} \theta
$$

In the case of a powder-like lamellar sample, the apparent diffusion coefficient is isotropic. Its value measured in any direction is:

$$
\langle D\rangle=\left(D_{/ /}+2 D_{\perp}\right) / 3
$$

On the other hand, for a $2 \mathrm{D}$ powder, the mean value of the diffusion coefficient measured in any direction perpendicular to the axis of symmetry is:

$$
\langle D\rangle=\left(D_{/ /}+D_{\perp}\right) / 2
$$

\subsubsection{Small-angle X-ray scattering}

SAXS experiments were performed with a laboratory setup that has already been described in detail. ${ }^{32}$ It consists of a rotating $\mathrm{Cu}$ anode generator (Rigaku), graded layer Ni/C mirror optics (Osmic), vacuum tubes (inserted between the sample and the detection), and a CCD camera (Princeton). 


\section{Results}

\subsection{Sample alignment}

The alignment of a lamellar phase in the NMR magnetic field can be monitored in-situ by following the deuterium NMR signal from $\mathrm{D}_{2} \mathrm{O}$ solvent molecules. Indeed, the ${ }^{2} \mathrm{H}$ NMR spectrum of an isotropic ("powder-like") distribution of $\mathrm{L}_{\alpha}$ phase domains has a characteristic shape that comes from the isotropic averaging of a Pake doublet. ${ }^{33}$ In an aligned $\mathrm{L}_{\alpha}$ phase, all the lamellae make the same angle with the magnetic field, and hence the ${ }^{2} \mathrm{H}$ NMR spectrum shows only a doublet. Finally, in the isotropic phase, all ${ }^{2} \mathrm{H}$ quadrupolar interactions are averaged out and the signal is just a single very thin peak.

A complex spectrum is observed when a new sample is first introduced in the spectrometer (fig. 2a,b), which reflects some partial alignment of the lamellar phase. This alignment is probably due to the shear that occurred when the NMR tube was filled. As the temperature is increased, this spectrum evolves to the familiar spectrum typical of an isotropic distribution of domains and the quadrupolar splitting $\left(\Delta v_{\mathrm{q}}\right)$ becomes smaller (fig. 2c). Near the transition temperature, an isotropic peak starts to grow and the powder $\mathrm{L}_{\alpha}$ phase disappears leaving at its place a small amount of aligned $\mathrm{L}_{\alpha}$ phase (fig. $2 \mathrm{~d}$ ). At even higher temperature, the sample becomes completely isotropic (fig. 2e). When the temperature is lowered, the isotropic phase gives place to an aligned $\mathrm{L}_{\alpha}$ phase (fig. $2 \mathrm{f}, \mathrm{g}$ ), which is characterized by a doublet of narrow lines.

The ${ }^{2} \mathrm{H}$ NMR spectra show that the lamellar phase is indeed aligned but the alignment direction was determined more directly by X-ray scattering. A $1 \mathrm{~mm}$ cylindrical X-ray capillary 
was filled with a doped $\mathrm{L}_{\alpha}$ phase sample and inserted into an NMR tube. Then, it was aligned according to the above procedure; its SAXS pattern can be seen in figure 3. The concentration of the X-ray scattering signal in the horizontal direction proves that all the lamellae are arranged parallel to the long axis of the capillary and that the "aligned" sample is actually a 2-dimensional distribution of lamellar domains with directors perpendicular to the magnetic field ("2D powder").

The quadrupolar splitting $\left(\Delta v_{\mathrm{q}}\right)$ of the ${ }^{2} \mathrm{H}$ doublet depends mostly on the tumbling dynamics of the water molecules at the interface. It is proportional to the average order parameter of the water O-D bonds. ${ }^{34,35}$ The quadrupolar splitting changes both with the surfactant volume fraction and the temperature of the sample. The variation of quadrupolar splitting with surfactant concentration at constant POM volume fraction is shown in figure $4 \mathrm{a}$. As expected, $\Delta v_{\mathrm{q}}$ increases when the water is more confined as it interacts more strongly with the bilayer. ${ }^{30}$ A similar variation, although to a lower extent, can be observed when the temperature of a sample is decreased (data not shown). It is well known that, at lower temperature, the hydrophilic heads of $\mathrm{C}_{\mathrm{m}} \mathrm{EO}_{\mathrm{n}}$ surfactants hydrate, and hence a greater fraction of water is bound to the bilayer. More unexpectedly, the quadrupolar splitting decreases with increasing POM concentration at constant surfactant volume fraction (fig. 4b). This might mean though that the POMs replace some of the structured water close to the interface.

The alignment procedure can also be followed by ${ }^{1} \mathrm{H}$ NMR as can be seen in figure 5 . Initially, the spectrum is composed of a broad peak $(50 \mathrm{~Hz})$ corresponding to the water molecules, and some featureless wide signal corresponding to the ether and alkyl components of the Brij 30 molecule. As the sample is heated to the isotropic phase, well-resolved signals are observed for the water, ether and alkyl components. Finally, as the temperature is decreased and 
an aligned $\mathrm{L}_{\alpha}$ phase is formed, the ether and alkyl signals disappear while the water signal remains narrow. Thus the formation of an aligned $\mathrm{L}_{\alpha}$ phase is accompanied with a large decrease of alkyl segment mobility, and, to a smaller extent, polyether segment mobility.

The ${ }^{31} \mathrm{P}$ NMR spectrum of a doped $\mathrm{L}_{\alpha}$ phase is shown in Supplementary Information (Figure S1). The central phosphorus atom gives a very narrow peak with a width of approximately $3 \mathrm{~Hz}$, which confirms the good alignment of the mesophase.

\subsection{Water diffusion in POM-doped $\mathrm{L}_{\alpha}$ phases}

The results of a PGSE-NMR water diffusion experiment on a $\phi_{\text {Surf }}=50 \%, \phi_{P O M}=3.5 \%$ oriented sample are shown in figure 6. As mentioned above, the sample is in the form of a 2D powder; the bilayers are arranged parallel to the z-axis but with a random orientation of the director in the $(x, y)$ plane. The diffusion coefficient of water was measured along the three space directions. It is immediately obvious that the water diffusion in the sample is anisotropic. The decay of the ${ }^{1} \mathrm{H}$ NMR signal is much faster when the gradient is applied along the $\mathrm{z}$ axis than when it is applied along the $\mathrm{x}$ or $\mathrm{y}$ axes (the decays are the same when the gradients are applied along the $\mathrm{x}$ and $\mathrm{y}$ axes). This is expected as diffusion along the $\mathrm{z}$ axis is parallel to the bilayers. The water molecules can move without obstacles in that direction, and thus diffuse fast. Moreover, the decay along the $\mathrm{z}$ axis is monoexponential, whereas the decay along the $\mathrm{x}$ axis (or the y axis) is not perfectly monoexponential. Again, this is related to the macroscopic anisotropy of the sample that is homogenous on a much larger lengthscale along the $\mathrm{z}$ axis than along the $\mathrm{x}$ axis. 
The same experiment was repeated with $\Delta$ increased to $500 \mathrm{~ms}$ (data not shown) in order to probe larger domains; the decays obtained for the shorter and longer diffusion times were exactly the same, evidencing the sample homogeneity. Therefore, the decay does not depend on the diffusion time $\Delta$. Moreover, for all samples, we checked that the decays along the $\mathrm{x}$ and the $\mathrm{y}$ axes are exactly the same. Thus, the samples are behaving like true 2D powders.

The monoexponential decays along the $\mathrm{z}$ axis direction for four samples with different surfactant volume fractions are shown in figure 7. They are faster for samples with smaller $\phi_{S u r f}$. On the other hand, samples with the same $\phi_{\text {Surf }}$ but different POM volume fractions present identical decays (fig. 8). Thus, the diffusion of water molecules in the POM-doped $\mathrm{L}_{\alpha}$ phase depends on the lamellar period (determined by $\phi_{S u r f}$ ), but not on the volume fraction of doping POMs.

To extract the water self-diffusion coefficients in the doped $\mathrm{L}_{\alpha}$ phase, the decays shown in the previous figures have been fitted by the Stejkal-Tanner equation (1) both along the $\mathrm{z}$ and the $\mathrm{x}$ axes. Because the equation only describes monoexponential decay, it is not strictly valid in the case of the $\mathrm{x}$ axis decay. However, the value extracted in this way remains close to the "true" value, which is given by the slope at the origin. The self-diffusion coefficient of water molecules in pure water at $305 \mathrm{~K}$ is $\mathrm{D}_{0}=2.8 \times 10^{-9} \mathrm{~m}^{2} \mathrm{~s}^{-1} \cdot 36,37$ Hereafter, all the measured water self-diffusion coefficients are normalized by this value.

In figure 9 , the normalized self-diffusion coefficient of water $\left(D_{w} / D_{0}\right)$ is plotted as a function of $\phi_{S u r f}$, at constant $\phi_{P O M}(3.5 \%)$. As qualitatively inferred from the decays, $\mathrm{D}_{\mathrm{w}}$ decreases with increasing $\phi_{\text {Surf }}$, from about $60 \%$ of its free water value at $\phi_{\text {Surf }}=40 \%$ to about $25 \%$ at $\phi_{\text {Surf }}=$ 70\%. The water molecules diffuse more slowly when the inter-lamellar space decreases. 
Furthermore, as expected, the diffusion coefficient along the $\mathrm{x}$ axis is about twice smaller in the whole range of volume fractions explored.

Figure 10 shows the evolution of $\mathrm{D}_{\mathrm{w}} / \mathrm{D}_{0}$ as a function of $\phi_{P O M}$, at constant $\phi_{\text {Surf }}(50 \%)$. This time, the diffusion coefficients do not vary with $\phi_{P O M}$, meaning that the POM volume fraction does not affect the diffusion of water. However, the diffusion along the $\mathrm{x}$ axis is still about twice slower for all samples.

Water diffusion was also studied in "true" single domains of the POM-doped $\mathrm{L}_{\alpha}$ phase in homeotropic orientation, with the director in the $(x, y)$ plane. For each measurement, $G_{x}$ and $G_{y}$ were varied so as to keep the gradient strength constant but to change its angle with respect to the fixed laboratory frame in $15^{\circ}$ steps. When the angle $\theta$ between the (fixed) director and the applied gradient approaches zero (respectively $90^{\circ}$ ), water diffusion perpendicular (resp. parallel) to the membranes is measured. This is illustrated in Figure 11 where the water diffusion coefficient in a POM-doped $\mathrm{L}_{\alpha}$ phase $\left(\phi_{\text {Surf }}=50 \%, \phi_{P O M}=3.5 \%\right)$ was measured as a function of $\theta$ and fitted by equation (2), thus yielding $D_{/ /}=(16 \pm 7) \times 10^{-12} \mathrm{~m}^{2} \mathrm{~s}^{-1}$ and $D_{\perp}=(13.7 \pm 0.3) \times 10^{-10}$ $\mathrm{m}^{2} \mathrm{~s}^{-1}$. The value of $D_{\perp}$ is in good agreement with the previous measurements and a very low value of $D_{/ /}$is thus measured, as intuitively expected.

\subsection{POM diffusion in doped $\mathrm{L}_{\alpha}$ phases}

The ${ }^{31} \mathrm{P}$ decay curves look very similar to the ${ }^{1} \mathrm{H}$ decay curves (Figure S2, Supplementary Information). Again, the decay along the $\mathrm{z}$ axis is monoexponential and fast, while the decay along the $\mathrm{x}$ axis is multiexponential and about twice slower (here also, the decays are the same for the $\mathrm{x}$ and $\mathrm{y}$ axes). This is expected because the measurements have been done successively on 
the same samples and the texture of the lamellar phase is exactly the same. The self-diffusion coefficients were extracted by fitting the decays with the Stejkal-Tanner equation as before.

The POM self-diffusion coefficient in a $\phi_{P O M}=5 \%$ aqueous solution was measured and used as a reference for normalization. Its value was found to be $4.6 \times 10^{-10} \mathrm{~m}^{2} \mathrm{~s}^{-1}$. For a spherical particle, this value gives a hydrodynamic radius of $0.48 \mathrm{~nm}$ which compares rather well with the actual radius of $0.55 \mathrm{~nm}$.

The normalized POM self-diffusion coefficients along the $\mathrm{z}$ axis are shown as a function of $\phi_{\text {Surf }}$, at constant $\phi_{P O M}(3.5 \%)$, in figure 12a. We note that the POM diffusion coefficient in the $\mathrm{L}_{\alpha}$ phase does not change dramatically when the phase becomes more concentrated in surfactant and that the POM diffusion coefficient in the lamellar phase is about $1 / 40$ of its value in solution.

The normalized POM self-diffusion coefficients are shown as a function of $\phi_{P O M}$, at constant $\phi_{\text {Surf }}(50 \%)$, in figure $12 \mathrm{~b}$. Surprisingly, the diffusion seems to be slower when the POM volume fraction increases, which might be due to mutual obstruction between the POMs.

4 Discussion

\subsection{POM self-diffusion}

We now discuss the evolution of the POM self-diffusion coefficients as a function of $\phi_{\text {Surf }}$. As the surfactant volume fraction increases, the particles are confined in a thinner water layer. The width of this layer is directly given by:

$$
d_{w}=\delta\left(1 / \phi_{\text {surf }}-1\right)
$$


where $\delta=3.4 \mathrm{~nm}$ is the bilayer width obtained by X-ray scattering. ${ }^{24}$ In our measurements, $d_{w}$ varies between 1-5 times the diameter of a POM. This variation was expected to have a strong effect on POM diffusion, as it is actually observed for the water molecules.

It has recently been shown that the diffusion of small spherical particles confined between surfactant bilayers can be adequately described by a simple model due to Faxen. ${ }^{38,39}$ In this model, a spherical particle diffusing between two parallel hard plates experiences an extra hydrodynamic friction force. This force is due to shearing the solvent between the particle that moves at some velocity and the wall that imposes zero velocity. The force depends on the distance between the particle and the plate. The particle self-diffusion coefficient is then reduced by a factor of:

$$
D / D_{0}=\left(1-2 \frac{R_{H}}{d_{w}}\right)
$$

where $D_{0}$ is bulk diffusivity, $R_{H}$ is the hydrodynamic radius of the particle, and $d_{\mathrm{w}}$ is the distance between the plates.

This model does not take into account either bilayer fluctuations or the fact that the bilayers are fluid, so the solvent can have a non-zero velocity component parallel to the interface. However, it has been shown to give correct predictions for the self-diffusion coefficient of small spherical particles of $R_{H} \approx 1 \mathrm{~nm}$ confined between $\mathrm{C}_{12} \mathrm{EO}_{5}$ bilayers, a situation very close to our own. ${ }^{39}$

In figure 13, the measured POM self-diffusion coefficients are compared to the predictions of the Faxen model for a spherical particle of radius $R_{H}=0.55 \mathrm{~nm}$. It is obvious that the model does not agree with the measured self-diffusion coefficients. The POMs diffuse much too slowly in the lamellar phase. 
The very low self-diffusion coefficients of the POMs and their non-dependence on the width of the water layer very probably indicate that they do not freely diffuse in the inter-bilayer space. This nicely confirms our previous interpretation of X-ray scattering measurements which suggested that the POMs are located close to the hydrophilic heads of the surfactant bilayer. ${ }^{24}$ The self-diffusion coefficient measurements are further proof of the existence of a strong POMbilayer interaction of unknown origin. This interaction is strong enough that it affects the dynamic properties of the POMs. In a way, their diffusion is linked to that of the surfactant molecules. The viscosity of a $\mathrm{C}_{12} \mathrm{EO}_{5}$ bilayer has been measured before, and found to be approximately $80 \mathrm{mPa} . \mathrm{s}$, or about 80 times that of water. ${ }^{39}$ This increase in viscosity has the right order of magnitude to explain the reduction of the POM self-diffusion coefficient to $1 / 40$ of its value in water. It is as if the POMs were mainly diffusing within the polyethylene oxide brush.

On the other hand, POM diffusion is clearly anisotropic. For a 2D powder sample, the selfdiffusion coefficient measured along the x-axis is the average value of $D_{/ /}$and $D_{\perp}$, the diffusion coefficients perpendicular and parallel to the $\mathrm{L}_{\alpha}$ phase director, respectively. The self-diffusion coefficient measured along the z-axis gives directly $D_{\perp}$. For all four samples, the self-diffusion coefficient is twice larger along the $\mathrm{z}$-axis than along the $\mathrm{x}$-axis, as expected for a 2D powder (Eq. 4). This means that $D_{/ /}$is negligible compared to $D_{\perp}$. The lamellar phase does not seem to contain any defects that would allow POM diffusion across the bilayers.

Moreover, the POM diffusion coefficient seems to decrease with increasing POM doping (figure 12b). Although the effect is quite weak, it is a surprising result because, at these rather low volume fractions $\left(\phi_{P O M} \approx 2-6 \%\right.$ ), a strong effect of the interaction between particles on their diffusion is unexpected. Indeed, PGSE-NMR experiments on aqueous POM solutions at different volume fractions (1-10\%), have given identical results for $\mathrm{D}_{\text {POM. However, the POMs are }}$ 
strongly attracted to the surfactant hydrophilic heads and are thus almost certainly confined in a layer thinner than the inter-bilayer distance. This confinement makes the apparent volume fraction close to the bilayers much higher than the nominal one, and interparticle interactions can therefore affect diffusion.

\subsection{Water self-diffusion}

The decrease of the water self-diffusion coefficient with increasing surfactant volume fraction is intuitive. When the aqueous layer is thinner (i.e. when $\phi_{\text {surf }}$ increases), the influence of the confining bilayers becomes more important, and so the diffusion coefficient decreases. However, the reduction of diffusion constant is much too large to be due to geometric confinement alone. Indeed, applying Faxen's formula (6) with a water hydrodynamic radius of $\sim 0.1 \mathrm{~nm}$ leads to a correction of only about $10 \%$ compared to free diffusion of water in bulk. Besides, the evolution of $\mathrm{D}_{\mathrm{w}}$ with $\phi_{\text {Surf }}$ is very different from that of $\mathrm{D}_{\mathrm{POM}}$ (Figure 9 and Figure 12a). This suggests that water diffusion is governed by a different mechanism than the adsorption process that controls POM diffusion and was discussed in the previous section.

The decrease in water diffusion coefficient may actually also be due to the existence of a fraction of surface-associated water molecules. Such molecules are not free to move between the bilayers, but are bound to the hydrophilic heads of the surfactant. As $\phi_{\text {Surf }}$ increases, the ratio of bilayer area to total water volume also increases, and hence there is a greater fraction of bound molecules. The observed diffusion coefficient, assuming fast exchange between the two environments, is the average of the diffusion coefficients of the free and of the surface-associated molecules. 
In a lamellar phase, a fraction, $f_{\text {sa }}$, of the water molecules are in strong interaction with the hydrophilic heads. This water is called "surface-associated" and its diffusion coefficient is much smaller than in the bulk. A very simple model $^{40}$ of diffusion in such a lamellar phase considers two regions with different diffusion coefficients:

$$
D=f_{s a} D_{s a}+\left(1-f_{s a}\right) D_{\text {free }}
$$

where $D_{s a}$ is the diffusion coefficient of the surface associated water. This equation is only valid for fast exchange, on the NMR timescale, between surface-associated molecules and free molecules. If that were not the case though, the PGSE decay would be biexponential, with two well-separated decays as the diffusion coefficients are very different.

By setting $D_{s a}=0, f_{s a}$ can be estimated for each $\phi_{S u r f}$ and therefore the number $\mathrm{n}_{\mathrm{b}}$ of water molecules bound per ethylene oxide segment. We obtain: $f_{s a} \sim 35 \%$ and $\mathrm{n}_{\mathrm{b}} \sim 2.8$ at $\phi_{\text {Surf }}=40 \%$, $f_{s a} \sim 55 \%$ and $\mathrm{n}_{\mathrm{b}} \sim 2.9$ at $\phi_{\text {Surf }}=50 \%, f_{\text {sa }} \sim 60 \%$ and $\mathrm{n}_{\mathrm{b}} \sim 2.1$ at $\phi_{\text {Surf }}=60 \%$, and $f_{\text {sa }} \sim 70 \%$ and $\mathrm{n}_{\mathrm{b}} \sim 1.6$ at $\phi_{\text {Surf }}=70 \%$. Such large fractions probably reflect the hydrogen bond network of confined water and the values of $n_{b}$ are in fair agreement with those published in the literature. $^{41,42}$

The results for samples with constant $\phi_{\text {Surf }}=50 \%$ and varying POM volume fraction (figure 10) show that POM doping has no influence on the water diffusion coefficient. This means that the structure of the $\mathrm{L}_{\alpha}$ phase is little affected by POM doping, which agrees well with the fact that the lamellar period remains unchanged. ${ }^{24}$

Finally, the PGSE-NMR measurements of the single-domain samples in homeotropic alignment showed a large water diffusion anisotropy, $\frac{D_{\perp}}{D_{/ /}}=85 \pm 30$, in good agreement with the anisotropy $(>72)$ measured by Wasterby in lipid bilayers. ${ }^{43}$ 
5 Conclusion

Anisotropic water self-diffusion in partially aligned (“2-D powder") samples of the POMdoped $\mathrm{L}_{\alpha}$ phase was studied as a function of surfactant and POM volume fractions. The diffusion coefficient parallel to the bilayers varies in the range $(6-17) \times 10^{-10} \mathrm{~m}^{2} \mathrm{~s}^{-1}$, depending on $\phi_{S u r f}$. This compares rather well with the diffusion coefficients found in other lamellar phase systems. ${ }^{35,44-46}$ Moreover, water diffusion does not depend on the POM volume fraction. Additional experiments with "true" single domains allowed us to directly measure the water diffusion anisotropy that is quite large but comparable to results previously reported in the literature. This suggests that POM-doping does not induce the formation of defects in the lamellar $\mathrm{L}_{\alpha}$ phase.

PGSE-NMR experiments with "2-D powder" samples have shown that the translational diffusion of the POMs in the $\mathrm{L}_{\alpha}$ phase is also quite anisotropic but is much slower than what would be expected on the basis of confinement alone. Furthermore, POM diffusion is not at all affected by the thickness of the aqueous medium separating the bilayers. Hence, the POMs do not freely diffuse in the inter-bilayer space, but strongly interact with the polyethylene oxide brushes that cover each side of the bilayers. This counter-intuitive but strong interaction of negatively charged POMs with the non-ionic surfactants could be mediated by $\mathrm{H}^{+}$cations that are very probably attracted by the ethylene oxide groups and that could in turn attract the POMs. ${ }^{47}$ This kind of information will prove important not only from a fundamental point of view, when trying to understand the interaction potential of the confined POMs, but also from a practical perspective, when formulating POM-doped hybrid systems comprised of non-ionic surfactants. 
Acknowledgements: We are deeply indebted to Paul Sotta and Mehdi Zeghal for very helpful discussions. A.S.Poulos gratefully acknowledges support from a Marie Curie action ( ${ }^{\circ}$-MESTCT-2004-514307) and from a Triangle de la Physique contract ( $N^{\circ}$ OTP 26784).

Supporting Information Available:

Figure $\mathrm{S} 1$ of the ${ }^{31} \mathrm{P}$ NMR spectrum of a doped $\mathrm{L}_{\alpha}$ phase.

Figure S2 of the ${ }^{31} \mathrm{P}$ PGSE-NMR decays.

This information is available free of charge via the Internet at http://pubs.acs.org/ 
(a) $\left(\right.$ b) ${ }_{\mathrm{H}, \mathrm{C}} \mathrm{N}[\mathrm{O}]_{4}^{\mathrm{OH}}$

(c) ${ }^{80}{ }^{10}$ 
Figure 1: (a) Representation of the $\left[\mathrm{PW}_{12} \mathrm{O}_{40}\right]^{3-}$ polyoxometalate anion. The central tetrahedron (shown in pink) has the phosphorus atom at its center. (b) Chemical formula of the non-ionic surfactant $\mathrm{C}_{12} \mathrm{EO}_{4}$, the main component of Brij 30. (c) Phase diagram (at room temperature) of the (Brij $30 / \mathrm{H}_{2} \mathrm{O} /\left[\mathrm{PW}_{12} \mathrm{O}_{40}\right]^{3-}$ ) hybrid system represented as a function of the overall surfactant volume fraction, $\phi_{S u r}$, and the POM volume fraction in the aqueous phase, $\phi_{P O M}$. The solid circles represent the samples of the $\mathrm{L}_{\alpha}$ phase that were studied by PGSE-NMR. 


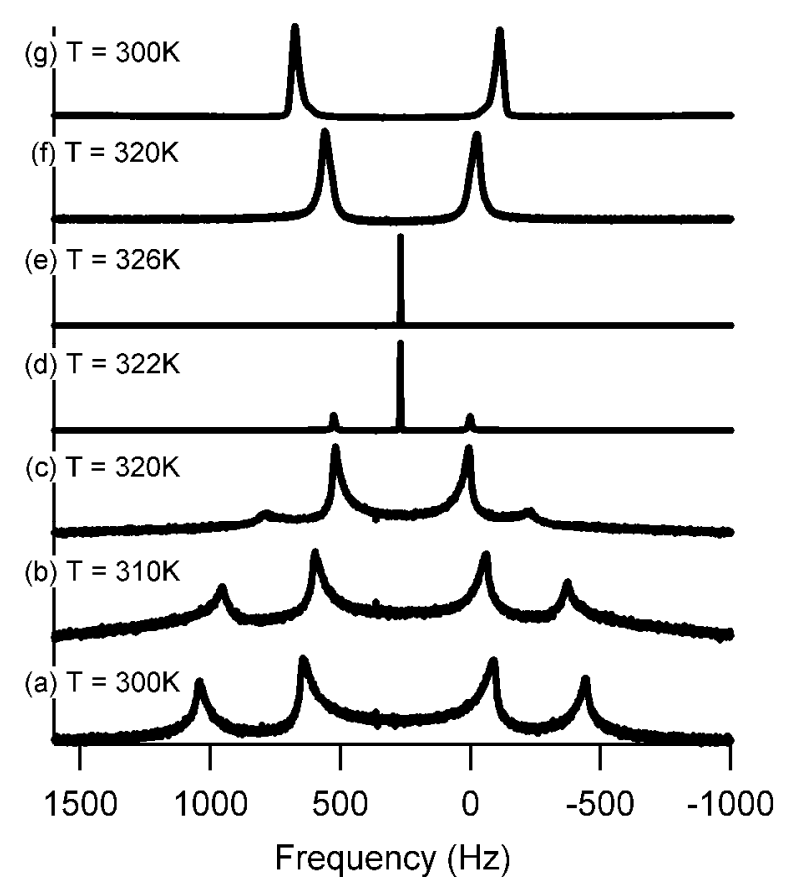

Figure 2: ${ }^{2} \mathrm{H}$ NMR spectra from $\mathrm{D}_{2} \mathrm{O}$ solvent molecules of a doped $\mathrm{L}_{\alpha}$ phase $\left(\phi_{\text {Surf }}=60 \%, \phi_{P O M}=\right.$ $3.5 \%$ ) at different temperatures. (a) Spectrum of a sample that has just been introduced into the spectrometer. (b, c) Evolution upon increasing temperature. (d) Isotropic phase along with some remaining aligned $L_{\alpha}$ phase. (e) Pure isotropic phase. (f,g) The temperature was decreased and an aligned $\mathrm{L}_{\alpha}$ phase was formed. 


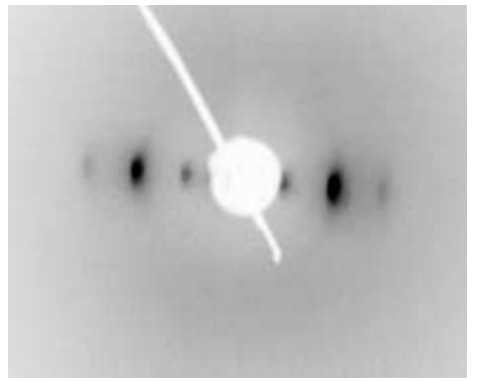

Figure 3: X-ray diffraction pattern showing the alignment of the lamellae parallel to the long axis of the capillary. (The capillary is very slightly tilted). 

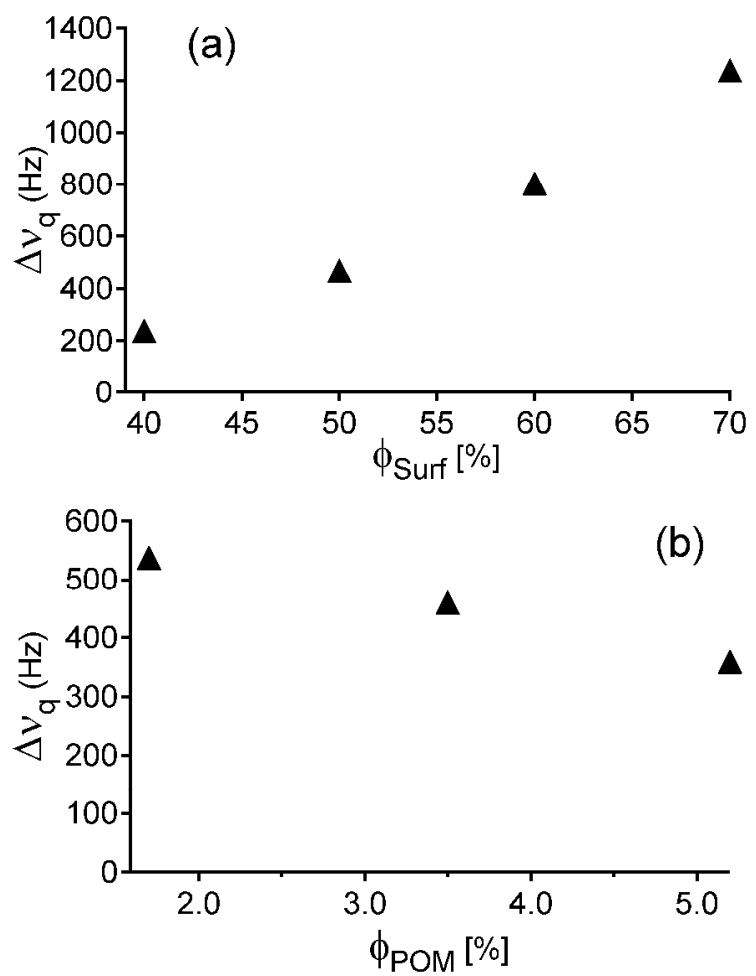

Figure 4: Quadrupolar splitting $\left(\Delta v_{\mathrm{q}}\right)$ of aligned lamellar phases (a) as a function of $\phi_{S u r f}$ at $\phi_{P O M}$ $=3.5 \%$, and (b) as a function of $\phi_{P O M}$ at $\phi_{S u r f}=50 \%$. (All measurements were performed at 310K.) 


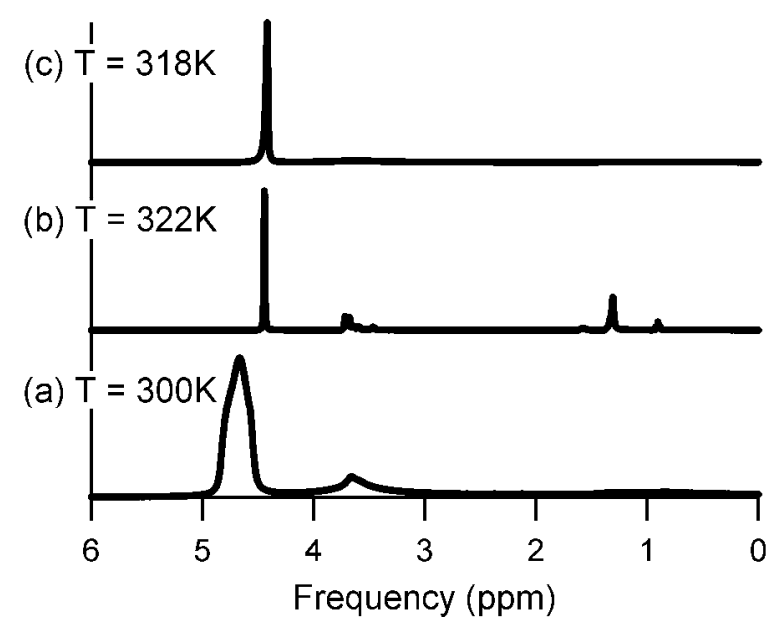

Figure 5: ${ }^{1} \mathrm{H}$ NMR spectra of a doped $\mathrm{L}_{\alpha}$ phase at different temperatures. (a) Powder-like $\mathrm{L}_{\alpha}$ phase. (b) Isotropic phase at high temperature. The water, ether, and alkyl peaks (located around $4.5,3.5$, and $1.5 \mathrm{ppm}$, respectively) are well resolved because of the high mobility of the phase. (c) Aligned $\mathrm{L}_{\alpha}$ phase. 


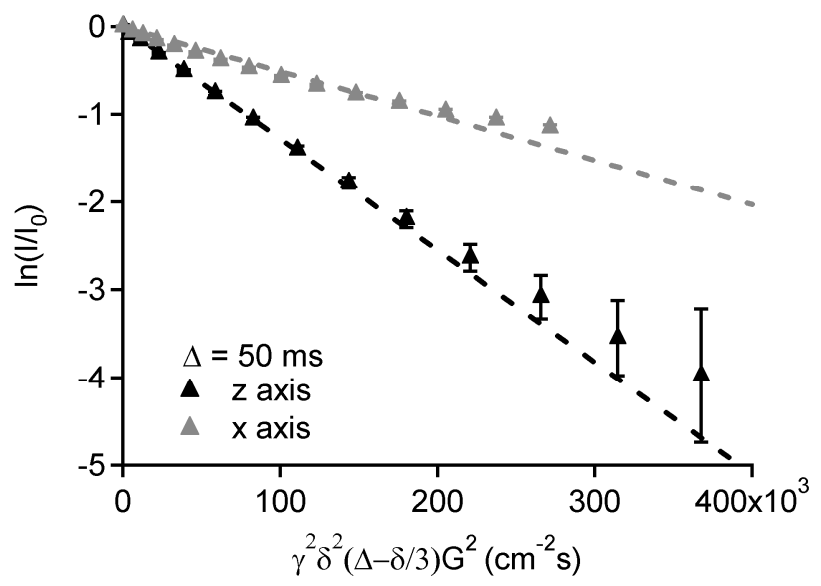

Figure 6: ${ }^{1} \mathrm{H}$ PGSE-NMR water-signal decays of an aligned sample with $\phi_{S u r f}=50 \%$ and $\phi_{P O M}=$ $3.5 \%$. The black triangles correspond to a gradient along the $\mathrm{z}$-axis direction, and the grey triangles along the $\mathrm{x}$ - or $\mathrm{y}$-axis directions (Diffusion time $\Delta=50 \mathrm{~ms}$ ). The diffusion anisotropy is clearly demonstrated by the change of gradient. The dotted lines are linear fits to the data using the Stejskal-Tanner equation (1). 


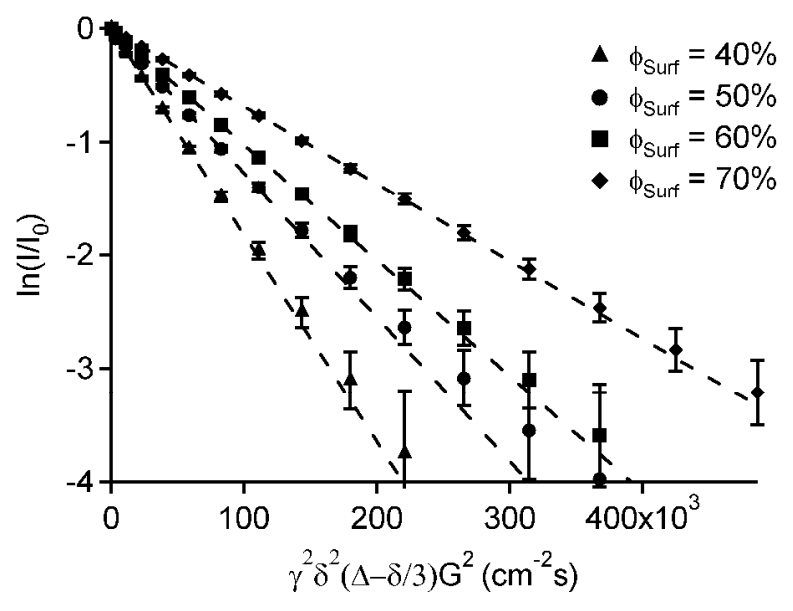

Figure 7: ${ }^{1} \mathrm{H}$ PGSE-NMR signal decays along the z-axis for samples with constant $\phi_{P O M}=3.5 \%$ and varying $\phi_{\text {Surf. }}$. The decay is monoexponential in all cases. A linear fit using equation (1) gives the water self-diffusion coefficient perpendicular to the director of the $\mathrm{L}_{\alpha}$ phase. 


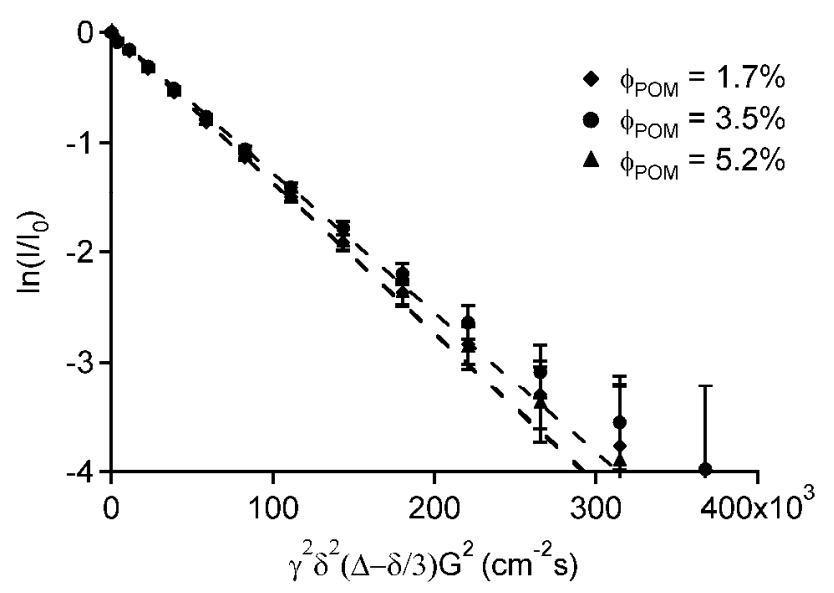

Figure 8: ${ }^{1} \mathrm{H}$ PGSE-NMR signal decays for samples with constant $\phi_{\text {Surf }}=50 \%$ and varying $\phi_{P O M}$. As expected, the self-diffusion coefficient of water does not change when the lamellar periodicity of the phase remains constant. 


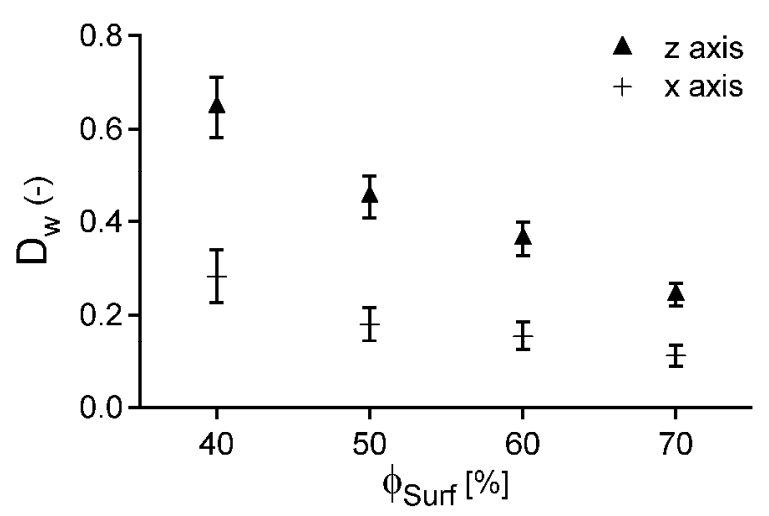

Figure 9: Variation of normalized water self-diffusion coefficients of aligned POM-doped lamellar phases with $\phi_{S u r f}$, at constant $\phi_{P O M}=3.5 \%$. The diffusion anisotropy can be clearly seen for all values of $\phi_{\operatorname{sur} f}$, moreover, it remains roughly constant. 


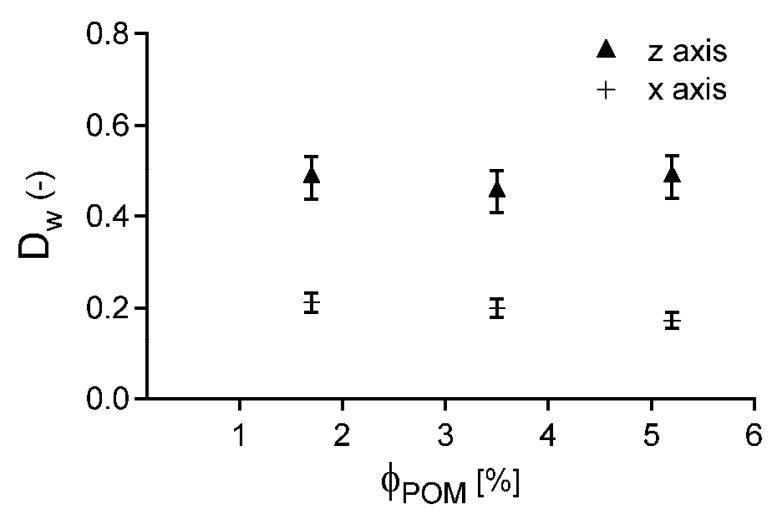

Figure 10: Variation of normalized water self-diffusion coefficients of aligned POM-doped lamellar phases with $\phi_{P O M}$, at constant $\phi_{S u r f}=50 \%$. The POM volume fraction does not affect water diffusion. 


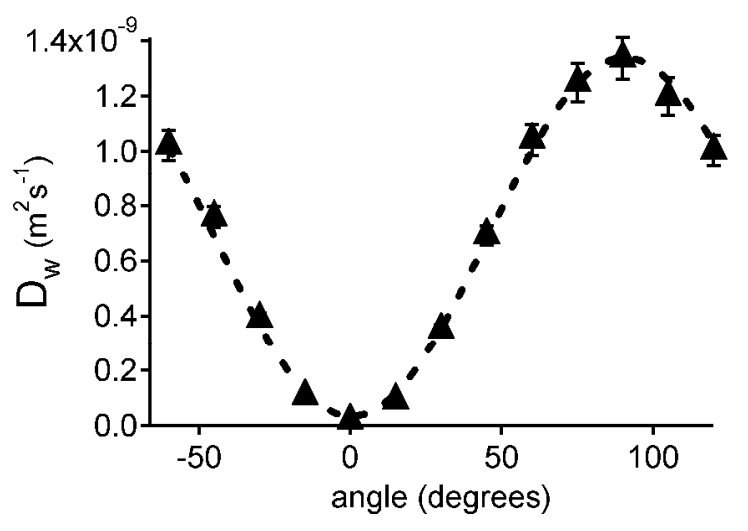

Figure 11: Angular dependence of the water diffusion coefficients in a single-domain of POMdoped $\mathrm{L}_{\alpha}$ phase $\left(\phi_{\text {Surf }}=50 \%, \phi_{P O M}=3.5 \%\right)$. The line is a fit to the data using equation (2). The minimum corresponds to $D_{/ /}$and the maximum to $D_{\perp}$. 

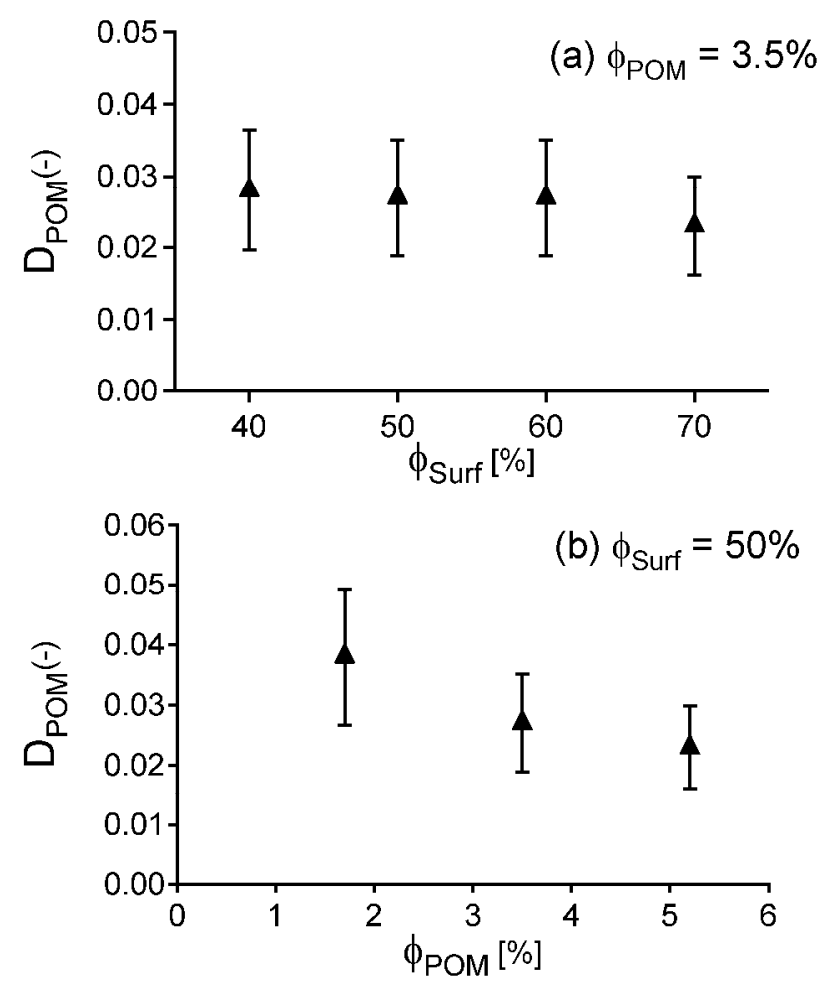

Figure 12: (a) Variation of normalized POM diffusion coefficients of aligned lamellar phases as a function of $\phi_{S u r f}$, at constant $\phi_{P O M}=3.5 \%$. (b) Variation of POM diffusion coefficients of aligned lamellar phases as a function of $\phi_{P O M}$, at constant $\phi_{\text {Surf }}=50 \%$. 


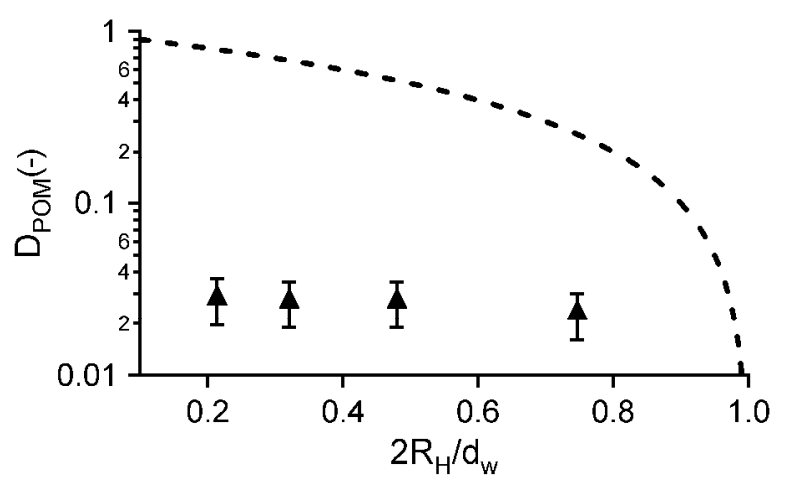

Figure 13: Comparison of Faxen equation predictions (dotted line) with the normalized POM diffusion coefficients measured along the z-axis. 
References

(1) Sanchez, C.; Soler-Illia, G. J. D. A.; Ribot, F.; Lalot, T.; Mayer, C. R.; Cabuil, V. Chemistry of Materials 2001, 13, 3061.

(2) Kickelbick, G. Angewandte Chemie-International Edition 2004, 43, 3102.

(3) Sanchez, C.; Arribart, H.; Guille, M. M. G. Nature Materials 2005, 4, 277.

(4) Beck, J. S.; Vartuli, J. C.; Roth, W. J.; Leonowicz, M. E.; Kresge, C. T.; Schmitt, K. D.; Chu, C. T. W.; Olson, D. H.; Sheppard, E. W.; Mccullen, S. B.; Higgins, J. B.; Schlenker, J. L. Journal of the American Chemical Society 1992, 114, 10834.

(5) Zhao, D. Y.; Feng, J. L.; Huo, Q. S.; Melosh, N.; Fredrickson, G. H.; Chmelka, B. F.; Stucky, G. D. Science 1998, 279, 548.

(6) Soler-illia, G. J. D.; Sanchez, C.; Lebeau, B.; Patarin, J. Chemical Reviews 2002, 102, 4093.

(7) Gouzerh, P.; Proust, A. Chemical Reviews 1998, 98, 77.

(8) Katsoulis, D. E. Chemical Reviews 1998, 98, 359.

(9) Pope, M. T.; Muller, A. Angewandte Chemie-International Edition in English $1991,30,34$.

(10) Freund, M. S.; Karp, C.; Lewis, N. S. Inorganica Chimica Acta 1995, $240,447$.

(11) Judeinstein, P.; Schmidt, H. J. Sol-Gel Sci. Tech. 1994, 3, 189.

(12) Polarz, S.; Smarsly, B.; Goltner, C.; Antonietti, M. Advanced Materials 2000, 12, 1503. 
(13) ClementeLeon, M.; Agricole, B.; Mingotaud, C.; GomezGarcia, C. J.; Coronado, E.; Delhaes, P. Langmuir 1997, 13, 2340.

(14) ClementeLeon, M.; Mingotaud, C.; Agricole, B.; GomezGarcia, C. J.; Coronado, E.; Delhaes, P. Angewandte Chemie-International Edition in English 1997, 36, 1114.

(15) Polarz, S.; Smarsly, B.; Antonietti, M. Chemphyschem 2001, 2, 457.

(16) Li, W.; Bu, W. F.; Li, H. L.; Wu, L. X.; Li, M. Chemical Communications 2005, 3785.

(17) Li, W.; Yi, S. Y.; Wu, Y. Q.; Wu, L. X. Journal of Physical Chemistry B 2006, $110,16961$.

(18) Li, W.; Yin, S. Y.; Wang, J. F.; Wu, L. X. Chemistry of Materials 2008, 20, 514.

(19) Nyman, M.; Ingersoll, D.; Singh, S.; Bonhomme, F.; Alam, T. M.; Brinker, C. J.; Rodriguez, M. A. Chemistry of Materials 2005, 17, 2885.

(20) Zhang, T. R.; Spitz, C.; Antonietti, M.; Faul, C. F. J. Chemistry-a European Journal 2005, 11, 1001.

(21) Moriguchi, I.; Orishikida, K.; Tokuyama, Y.; Watabe, H.; Kagawa, S.; Teraoka, Y. Chemistry of Materials 2001, 13, 2430.

(22) Bu, W. F.; Uchida, S.; Mizuno, N. Angewandte Chemie-International Edition 2009, 48, 8281 .

(23) Pradeep, C. P.; Misdrahi, M. F.; Li, F. Y.; Zhang, J.; Xu, L.; Long, D. L.; Liu, T.; Cronin, L. Angewandte Chemie-International Edition 2009, 48, 8309.

(24) Poulos, A. S.; Constantin, D.; Davidson, P.; Imperor, M.; Pansu, B.; Panine, P.; Nicole, L.; Sanchez, C. Langmuir 2008, 24, 6285.

(25) Stejskal, E. O.; Tanner, J. E. Journal of Chemical Physics 1965, 42, 288. 
(26) Callaghan, P. T.; Legros, M. A.; Pinder, D. N. Journal of Chemical Physics 1983, $79,6372$.

(27) Strey, R. Berichte Der Bunsen-Gesellschaft-Physical Chemistry Chemical Physics 1996, $100,182$.

(28) Marosi, L.; Platero, E. E.; Cifre, J.; Arean, C. O. Journal of Materials Chemistry 2000, 10, 1949.

(29) Pope, M. T. Heteropoly and isopolyoxometalates; Springer: New York, 1983.

(30) Briganti, G.; Segre, A. L.; Capitani, D.; Casieri, C.; La Mesa, C. Journal of Physical Chemistry B 1999, 103, 825.

(31) Altieri, A. S.; Hinton, D. P.; Byrd, R. A. Journal of the American Chemical Society 1995, 117, 7566.

(32) Imperor-Clerc, M.; Davidson, P. European Physical Journal B 1999, 9, 93.

(33) Pake, G. E. The Journal of Chemical Physics 1948, 16, 327.

(34) Pelletier, O.; Sotta, P.; Davidson, P. Journal of Physical Chemistry B 1999, 103, 5427.

(35) Yethiraj, A.; Capitani, D.; Burlinson, N. E.; Burnell, E. E. Langmuir 2005, 21, 3311.

(36) Holz, M.; Heil, S. R.; Sacco, A. Physical Chemistry Chemical Physics 2000, 2, 4740.

(37) Mills, R. Journal of Physical Chemistry 1973, 77, 685.

(38) Faxen, H. Ann. Phys. 1922, 4, 89.

(39) Moreau, P.; van Effenterre, D.; Navailles, L.; Nallet, F.; Roux, D. European Physical Journal E 2008, 26, 225. 
(40) Holmes, M. C.; Sotta, P.; Hendrikx, Y.; Deloche, B. Journal De Physique Ii 1993, 3, 1735 .

(41) Romsted, L. S.; Yao, J. H. Langmuir 1996, 12, 2425.

(42) Sterpone, F.; Pierleoni, C.; Briganti, G.; Marchi, M. Langmuir 2004, 20, 4311.

(43) Wasterby, P.; Oradd, G.; Lindblom, G. Journal of Magnetic Resonance 2002, 157, 156.

(44) Blinc, R.; Easwaran, K.; Pirs, J.; Volfan, M.; Zupancic, I. Physical Review Letters 1970, 25, 1327.

(45) Gaemers, S.; Bax, A. Journal of the American Chemical Society 2001, 123, 12343.

(46) Tiddy, G. J. T. Journal of the Chemical Society, Faraday Transactions 1 1977, 73, 1731.

(47) Alexandridis, P.; Holzwarth, J. F. Langmuir 1997, 13, 6074. 